\title{
Pressure distribution and cavitation in counter- vortex flow energy dissipators of hydraulic spillways
}

\author{
Genrikh Orekhov*
}

Moscow State University of Civil Engineering, Yaroslavskoe shosse, 26, Moscow, 129337, Russia

\begin{abstract}
The article is devoted to the study of cavitation phenomena of counter-vortex flow energy dissipators that can be used in hydraulic spillways. The spillways providing the surface flow transitionn at hydraulic structures are equipped with energy dissipators of the discharged flow. An increase in the effective pressure on the hydropower project leads to an increase in the flow velocities and, hence, to an increase in the loads acting on the structures. One of such a manifestation is cavitation and cavitation erosion associated with it, which can lead to destruction of structures. The objective of the study consists in determining the cavitation characteristics of counter-vortex flow energy dissipators. The study was carried out by modeling using high-head physical models. The countervortex method of excess flow energy dissipation based on the work of viscous friction forces allows the flow energy to be dissipated in a very short part of the flow conductor system of the spillway. This feature of the counter-vortex flow energy dissipator imposes special requirements to the study of cavitation phenomena. The carried out studies resulted in obtaining the distribution of pressures lengthwise the flow conductor system of the energy dissipator with spiral swirls. The values of the cavitation coefficient and relative pressure at different points of the device are given. In the conclusions it is noted that the most dangerous part from the viewpoint of cavitation orrurence is the initial section of the flow energy dissipation chamber; cavitation due to flow separation and bubble cavitation occur within the flow and does not affect the structural elements; on a large-scale model working for 500 hours at pressures of up to $70 \mathrm{~m}$ cavitation erosion of the walls has not been detectd.
\end{abstract}

\section{Introduction}

Spillways are the integral part of hydraulic and hydropower facilities. They are used both during construction and operation periods. The type, composition and layout of the spillway system in the hydraulic facilities depends on the design flow rate, the difference in the levels of the pools, the purpose and type of the hydro system, the topographic and geological conditions of the site and the operation requirements. Considering the hydraulic, structural and layout features, there are several basic schemes for discharging water downstream:

\footnotetext{
* Corresponding author: orehov_genrih@ @mail.ru
} 
surface free-flow [1, 2], pressure submerged or bottom [3, 4], open and closed, integrated with a dam and shore spillways [5].

For flow transition from the upper pool to the tail water a dissipator of the kinetic energy of the discharged stream is required. In practice, the most commonly applied are stilling basins $[6,7]$, energy dissipation by jet throwing from hydraulic structures towards the tail water area $[8,9]$. In some cases, depending on water flow velocity, topography, geological features of the spillway foundation, a stepped surface spillway design is applied or that in the form of various piers located in a certain order [10]. Such designs of the flow conductor system of the spillway serves at the same time a flow energy dissipator at the entire length of the spillway [11].

In interaction of the flow with structural elements significant hydrodynamic loads arise transmitted to the structural elements of the spillway and, in some cases, to other structures of the hydropower complex. Moreover, as a result of the effect of high-velocity flows on the structures, cavitation manifestation and associated cavitation erosion can be observed $[12,13,14]$. In addition, such phenomena as aeration [15], wave formation and other ones can occur. Such manifestations are all the more intensive, the higher is the head on the structure and the flow velocity. Underestimation of these factors can lead to serious destruction of structural elements of the spillway and tailrace [16].

The transition to high heads requires new approaches to the study of the phenomena associated with high-velocity streams and development of fundamentally new types of currents and designs of the energy dissipators of flows to be discharged This approach forms new technological solutions in hydraulic engineering. One of them is the method of flow energy dissipation by means of counter-vortex dissipators based on of viscous friction forces of oppositely directed circulation currents [17]. This feature of the counter-vortex dissipator imposes special requirement on the study of cavitation phenomena taking place during its operation.

\section{Objective}

The research is aimed to study the cavitation phenomena that arise during operation of the counter-vortex flow energy dissiparor. Operation of such a dissipator is characterized by the flows with very high values of velocity gradients and, as a consequence, the formation of areas featuring the pressure below the atmospheric one. Since cavitation occurs in the areas of fluid flow with a reduced pressure (up to a value less than the pressure of saturated water vapor), it is important to know the distribution of pressure lengthwise the flow conductor of the counter-vortex dissipator.

The objectives of the study are as follows:

- experimental model studies of the distribution of pressures lengthwise the flow conductor system of the counter-vortex flow kinetic energy dissipator for high-head hydraulic spillway systems;

- determination in the flow path of areas with a pressure below atmospheric pressure;

- determination of the coefficient of cavitation and its dependence on the acting head;

- experimental assessment of the cavitation effect on the walls of the flow path of the dissipator.

\section{Method}

Spatial non-uniform flows with interacting, oppositely rotating, coaxially arranged layers of liquid or gas are formed in the counter-vortex flow energy dissipator. Such flows are not encountered in nature contrary to the longitudinal and circulation-longitudinal currents 
widely spread in nature and technology. They are characterized by a complicated form of distribution of velocity components and specific structural parameters. Such hydrodynamic structures of fluid (or gas) flows are formed due to a special organization of the initial longitudinal-axial flows. Therefore, practically the only method for solving essentially any problems of the dynamics of such flows is a physical experiment.

The studies of cavitation phenomena and associated pressure distributions in the flow path of the flow energy dissipator were carried out on a physical model. Figure 1 shows an experimental installation and Fig. 2 is a diagram of the flow conductor part of the countervortex flow energy dissipator incorporated in the experimental installation.

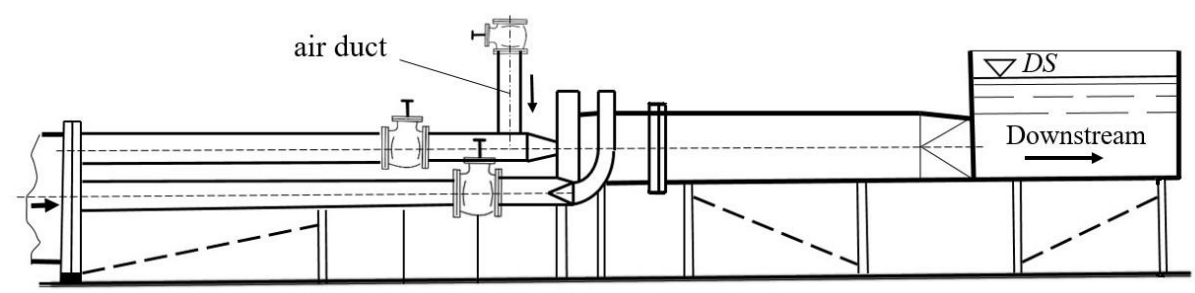

Fig.1. Experimental stand for cavitation studies

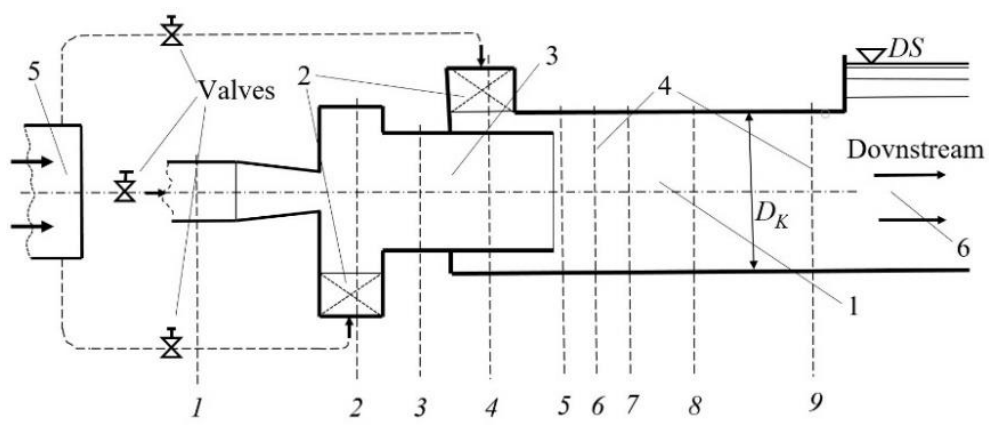

Fig. 2. Schematic drawing off the flow conductor of the counter-vortex flow energy dissipator with local swirls in the form of spiral cases (longitudinal section): 1 - cylindrical chamber for dissipation of kinetic energy of the flow, 2 - local swirls in the form of spiral cases, 3 - intermediate cylindrical branch pipe, 4 - measuring sections for measuring pressure, 5 - supply pressure pipeline, 6 - downstream.

The geometric and hydraulic characteristics of the model are as follows: the model length is $9.6 \mathrm{~m}$, the energy dissipation chamber diameter is $0.8 \mathrm{~m}$, the diameter of the intermediate branch pipe is $0.66 \mathrm{~m}$, the length of the energy diaaipation chamber is $5.6 \mathrm{~m}$, the length of the intermediate branch pipe is $1.8 \mathrm{~m}$, the local swirls are made in the form of a spiral caser with an enveloping angle of 360 degrees, the maximum operating head is $70 \mathrm{~m}$; the maximum flow rate is up to $3 \mathrm{~m}^{3} / \mathrm{s}$. The pressure on the walls of the flow energy dissipator conductor was measured by measuring instruments located in the sections shown in Fig. 2. The instruments in the measuring sections were located circumferentially the cylindrical surface of the flow energy dissipator elements. The pressure value in the measuring section was averaged according to the indications of several instruments.

The following values have been measured in the process of studies: $Q$ - flow rate passed through the model in different modes, $H$ - head, $P$ - pressure on the walls of the flow conductor portion. The mean square errors [18] of the measured values are: head, $H-$ $+/-3.3 \times 10^{-2}$, flow rate, $Q-+/-3.8 \times 10^{-2}$, pressure, $P-+/-3.6 \times 10^{-2}$. 


\section{Results}

The presence of high flow velocities, in which the counter-vortex flow energy dissipators operate, predetermines the need to predict of cavitation phenomena. Since the countervortex flow energy dissipator is based on interaction of swirled flows [19, 20], for which characteristic is the separation into high and low pressure zones, the basis for the analysis of cavitation conditions should be a picture of static pressures in the flow conductor portion of the flow energy dissipator that reveals low-pressure zones. It is also important to know the hydrostatic load on the elements of the flow conductor portion of the dissipator on the flow side

The curves in Figures 3 and 4 show the distribution of the relative averaged pressure on the walls lengthwise the flow conductor corresponding to the measuring sections shown in Fig. 2. The relative pressure was determined from expression

$$
\chi=\frac{P_{i}}{\rho g H}(1),
$$

where $P i$ is the pressure at the selected points of the measuring sections shown in Fig. 2, $\rho$ - water density, $g$ - acceleration of gravity, $H$ - acting head.

Fig. 3 shows the experimentally obtained distributions of the averaged relative pressures on the walls of the flow conductor of the counter-vortex flow energy dissipator depending on the acting head for its different parts: inner and outer chambers, intermediate branch pipes and dissipation chamber.

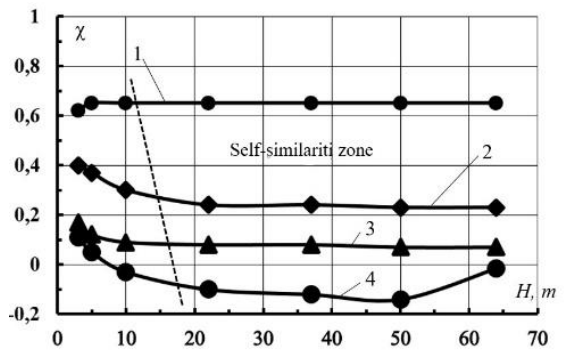

Fig. 3. Relationship $\chi=f(H)$, mode M.1-0-1. 1 - inner chamber, 2 - outer chamber, 3 - intermediate branch pipe, 4 - flow energy dissipation chamber

Adopted for calculations were the average values of relative pressure $\chi$ in the points located in the self-similarity zone of $\chi=f(H)$ relationship within the head range from 1 to $70 \mathrm{~m}$. Fig. 4 shows relationship $\chi=f(L)$, where $L$ is the length of the entire flow conductor portion of the counter-vortex flow energy dissipator. In process of model testing the following symbols have been adopted designating the operation mode of the device. " $M$ "designates "mode". Three figures following " $M$ "designate the operation of all the three supply pipelines. In case of closing of any pipeline " 0 " was entered in the designation instead of unity. In the modes with air supply symbol " $A$ " was entered in the designation. For example, the mode with three operating water conduits without air supply to the system was designated as M.1-1-1.The operation mode with the air supplied through the air duct (Fig. 1 ) and axial jet $-M \cdot 1-1 A-1$. The operation mode of the flow energy dissipator without axial water stream with the air supplied through air duct (Fig. 1) - M.1-A-1. The operation mode without air and axial jet supply $-M .1-0-1$. 


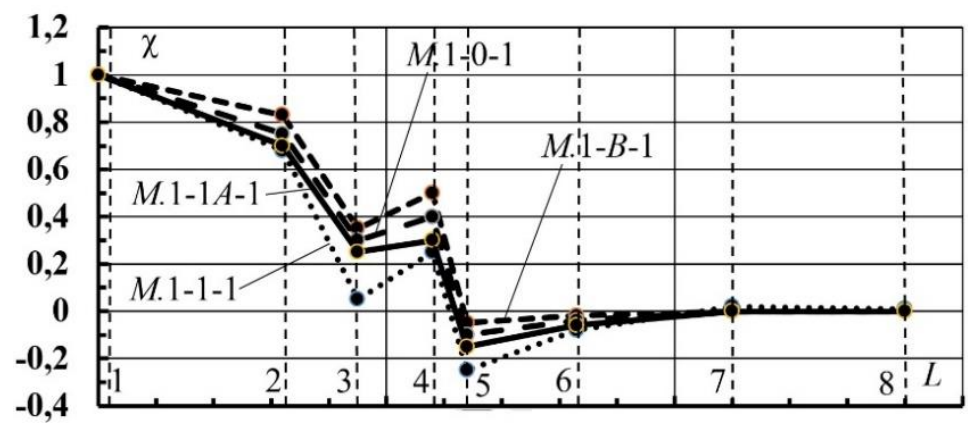

Fig. 4. Distribution of relative averaged pressure $\chi=P / \rho g H$ lengthwise the flow conductor $L$

The greatest relative hydrostatic pressure takes place in the swirl of the internal swirled flow (Fig. 3) and is $65 \%$ of $H$ for all three models in different modes of operation of the device. The greatest relative pressure is observed in operation modes with two water pipelines, than in the modes with three operating pipelines.

The pressure drop observed in the intermediate branch pipe and in the outer swirl in operation modes with three streams as compared with the modes with two operating pipelines can be explained as follows. The axial jet supplied to the central area in the zone of reduced pressure expands under the action of tensile forces of centrifugal mass forces, occupying a larger place than a compact axial jet. This result in a partial interaction along the periphery of the two streams just in front of the flow energy dissipation chamber. Moreover, the jet occupying a certain volume inside the steam-air core, reduces the thickness of the rotating layer of liquid at the wall. This leads to convergence of the integration limits in expression

$$
\rho \int_{r_{0}}^{R} \frac{u_{\theta}^{2}}{r} d r,
$$

where $u_{\theta}^{2}$-azimuthal (tangential) component of the total flow velocity vector in the energy dissipation chamber, $r$ - current radius, $r_{0}=D / 2$.

The change in the integration range in expression (2) indicates a decrease in pressure at the wall of the intermediate branch pipe. In the $M \cdot 1-1 A-1$ mode the effect of the flow of water flowing through the central pipeline increases. In this case, the thickness of the rotating layer decreases due to the elimination of the steam-air core in the axial area.

The main condition for normal operation of the flow energy dissipator consists in total dissipation of the energy-bearing azimuth (tangential) velocities of two interacting circulation flows. If this condition is met at the initial portion of the energy dissipation chamber, the pressure on the walls is mainly determined by the position of the tailwater level that is confirmed in $M .1-0-1$ or $M .1-A-1$ modes. Some reduction in pressure in such modes is possible due to external flow separation with its sharp turn by 90 degrees on the sharp edge connecting the local swirl of the external flow and the flow energy dissipation chamber (Fig. 2). In this case, the pressure value will depend on the head and will be about $5 \%$ of $H$ on the average.

For the modes with three operating pipelines $M .1-1-1$ the flow energy dissipation at the initial portion of the dissipation chamber is $(5 \div 10) \%$ of $H$ on the average. A greater value of negative pressures is observed in modes $M .1-1 A-1$. This is explained by ejecting effect of energy-intensive axial flow, which, when introduced in the zone of energy dissipation of two swirled flows, causes ejection at the initial sections of the flow in the energy dissipa- 
tion chamber. As the central axial flow disintegrates (this happens at a distance of (1-2) $D_{K}$ ) its influence on the flow pattern decreases. With the air supplied simultaneously with the axial flow the energy dissipation intensity decreases and the negative pressure values (vacuum) increase.

During the tests, the effect of the length of the streamlined unit on pressure distribution on the dissipation chamber walls was studied. Fig. 5,a shows the change in the relative pressure in the energy dissipation chamber with a streamlined unit and without it.

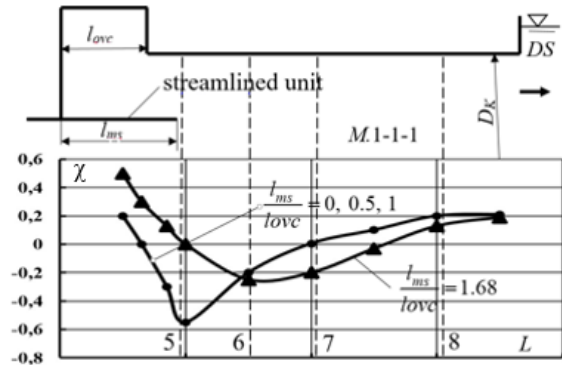

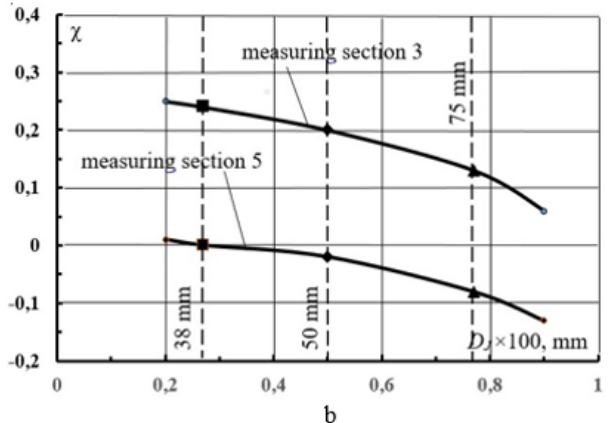

Fig. 5. a - effect of length of the intermediate fairing on distribution of relative pressure lengthwise the flow energy dissipation chamber, $b$ - effect of the diameter of central axial flow on pressure distribution in the intermediate branch pipe in the energy dissipation chamber.

When the device with a streamline unit, whose length is greater than the width of the external swirl, the value of the relative negative pressure decreases slightly from $5 \% \mathrm{H}$ to $3 \% \mathrm{H}$. Taking the above into account, the presence of a pressure reduction zone does not cause anxiety for similar swirls featuring high value of effective dimensionless geometric characteristics equal to $A=23.34$. The geometric characteristics [21] for a local swirl of tangential spiral single-swirl type was determined by formula

$$
A=\frac{\pi R R_{0}}{\delta S_{T}}
$$

where $R$ - radius of cylindrical channel, $R_{0}$ - effective radius, $\delta$ - jet compression factor, $S_{T}$ - area of the supply channel.

The effect of the diameter of the axial water conduit on the relative pressure distribution along the length of the flow conductor was studied (Fig. 5, b). This effect has been mainly revealed just in two places: in the intermediate branch pipe and at the initial portion of the energy dissipation chamber to measuring section 2 . With the increase in the area of the live section of the axial flow the pressure decreases: from $25 \%$ to $15 \%$ in the intermediate pipe and from $5 \%$ to $10 \%$ in the energy dissipation chamber. The presence of negative pressure areas in the flow conductor part of the counter-vortex energy dissipator - inside the swirled flows at the length of the intermediate branch pipe and in the initial portion of the chamber - raises a question of predicting the possibility of cavitation phenomena in different modes of the facility.

The following are the types of cavitation, which can be observed in the counter-vortex flow energy dissipator:

1. Within the zone of intensive flow energy dissipation with negative pressure in the steam-air core. Here, there is a cavitation due to flow separation (vortex) mainly occurring in vortices, at the boundary of shear of swirled currents (areas with high gradients of flow velocities tending to infinity $[22,23,24])$, and also at the boundary of a submerged axial jet 
in operation modes $M .1-1-1$ or M.1-1A-1 and internal swirled current. The intensity of the cavitation manifestations will be the higher the deeper vacuum forms in the axial zone of the flow. In modes $M .1-0-1$ and $M .1-A-1$ vortex cavitation will be generated mainly in the boundary zone of interaction of the external and internal swirled currents, that is, inside the flow and not close to the walls of the chamber. In process of studies this type of cavitation began manifesting at pressures $H \geq 20-25 \mathrm{~m}$. Vortex cavitation does not cause cavitation erosion of the elements of the flow conductor part of the dissipator.

2. Bubble form of cavitation, due to the presence of pulsation pressure, can be observed in the second cavitation-prone zone - the initial portion of the energy dissipation chamber. This form can cause cavitation erosion. In our case, there are two ways to prevent cavitation erosion. Air supply to the areas of low pressure in the initial portion of the energy dissipation chamber or by underflooding from the downstream side. In the experiments the air supply to the chamber corresponded to operation mode $M .1-1 A-1$.

Two parameters characterizing the cavitation phenomena have been studied: cavitation number and Thoma's criterion determined as

$$
K_{c a t}=\frac{p-p_{\text {sat }}}{0.5 \rho V^{2}} .
$$

Here $p$-absolute pressure in the measuring point, $p_{\text {sat }}$ - water-pour saturated at a temperature of $20^{\circ} \mathrm{C}, V^{2}$ - flow velocity in the measuring point. Calculation of the Thoma's criterion is made for its comparison with a certain critical number $K_{\text {cat.cyt }}$, when cavitation starts developing.

$$
K_{\text {cat }} \leq K_{\text {cat.cr }} .
$$

Under condition with $K_{\text {cat }}>K_{\text {cat.cyt }}$ there is no cavitation, with $K_{\text {cat }} \leq K_{\text {cat.cyt }}$ it occurs. For preliminary assessment the value $K_{\text {cat.cyt }}=1$ was chosen for irregularities located on flat walls. Fig. 6 shows the results of determination of the Thoma's criterion for the heads of 20, 40 and $60 \mathrm{~m}$ varying as a power function and with $H=80 \mathrm{~m}=1.0$, which means that at this head onset of cavitation conditions can be expected in the fifth measuring section of the flow energy dissipation chamber.

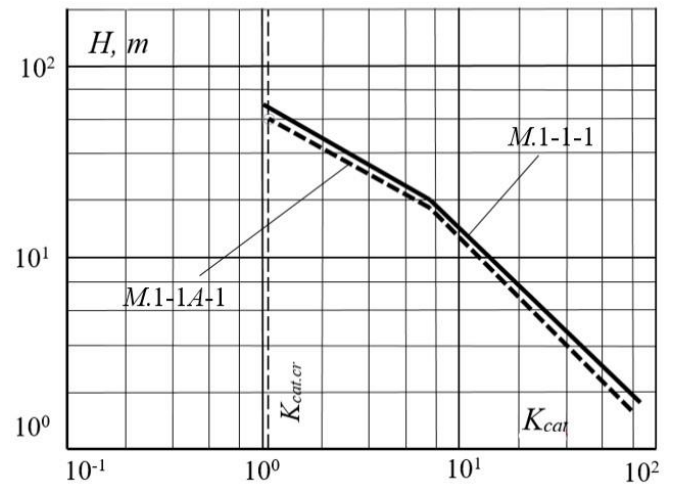

Fig. 6. Cavitation number - head relation curves under M.1-1A-1 operation mode

To identify the prevailing cavitation forms during the test the model worked for about 500 hours under cavitation mode. Metal surfaces of the flow conductor portion of the flow energy dissipator were preliminarily covered with a special varnish in cavitation-prone 
places. Visual examination of the internal surface of the dissipator did not revealed any cavitation erosion. This allows us to conclude that in the counter-vortex flow the cavitation phenomena mainly appear inside the flow and do not affect the walls of the device.

\section{Conclusions}

1. The magnitude of the relative pressure along the length of the flow-conductor portion of the counter-vortex flow energy dissipator varies non-uniformly. The most dangerous from the point of view of occurrence of cavitation phenomena is the initial section of the quench chamber. Here flow regions with negative pressure values (vacuum) are formed at a length on the order of two diameters of the dissipator.

2. The working process of high-pressure counter-vortex energy dissipators for hydraulic spillway systems is characterized by the presence of developed cavitation phenomena due to formation of low-pressure zones and extremely high velocity gradients at the boundary between interacting flows. The source of strong cavitation in the zone of direct contact of oppositely swirled flows is located inside the current and does not close on the walls of the device. This circumstance makes it possible to positively assess the operation of the counter-vortex dissipator structures from the point of view of cavitation erosion effect on them. 3. The dependence of cavitation coefficient on the head is a power mode. With increase of the effective head the cavitation coefficient decreases and with a head $H$ of about $80 \mathrm{~m}$, reaches a critical value equal to unity. Onset of cavitation can be expected with this head.

4. Long-term cavitation tests of the model showed that the cavitation attack did not close to the walls of the dissipator flow-conductor portion. On the experimental model, which worked for 500 hours at heads up to $70 \mathrm{~m}$, no cavitation erosion on the walls has been detected.

\section{References}

1. N. Fehn, D. Kozlov, I. Rumyancev. Power Techn. and Engin. 8 29-36 (2015)

2. A. Bayon, J. Toro, F. A. Bombardelli, J. Matos, P. López-Jiménez. J. of Hydroenvironment Research. 19 137-149 (2017)

3. R. Shemshi, A. Kabiri-Samani, J. of Hydraulic Research 55 248-258 (2017)

4. F. Wang, S. Wu, W. Wang, J. of Hydroelectric Eng. 36 65-73 (2017)

5. M. Mahzari, A.Schleiss, Dam. Eng. 20 (4) 307-327 (2010)

6. A. Gurjev, N. Khanov, N. Volgin. Scientific practical j. of environmental management 4 48-53 (2015)

7. G. Sudolskiy, Power Techn. and Eng. 8 32-40 (2016)

8. J.-h. WU, L. YAO, F. MA, W.-w. WU. J. of Hydrodynamics, Ser. B, 26, 86-93 (2014)

9. F. MA, Z. XU, J.-h. WU. J. of Hydrodynamics, Ser. B, 27 907-912 (2015)

10. J. Toro, F. Bombardelli, J. Paik, I. Meireles, A. Amador, Environmental Fluid Mechanics 16(6) 1195-1221 (2016)

11. A. Parsaie, A. Haghiabi, M. Saneie, H Torabi J. of Hydraulic Eng. 22(3) 281-292 (2016)

12. P. Zamankhan, Matematical Prob. in Eng., 2015, ID 872573 (2015)

13. S.Terrier, M. Pfister, A. Schleps, IAHR World Congress (2015)

14. M. Cihan Aydin, J. of Hydraulic Eng. 24 330-336 (2018)

15. T.Lui, J. Yang, J. Eng. Applications of Computational Fluid Mechanics 8 104-115, (2014)

16. I. Sliva, G. Lapin, Power Techn. and Engin. 11 44-51 (2017)

17. V. Volshanik, A. Zuikov, G. Orekhov, P. Churin, Power Techn. and Engin. 4 51-56 (2013) 
18. GOST 8.009-84. State system for ensuring the uniformity of measurements. Standardized metrological characteristics of measuring instruments (Standardinform, Moskov, $27 \mathrm{pp}, 2006)$

19. V. Volshanic, G.Orekhov, Vestnik MGSU 7 87-104 (2015)

20. V. Akhmetov, V. Volshanic, A. Zuikov, G. Orekhov, Simulation and Calculation of Counter-Rotating Flows (Publ. MISI-MGSU, Moscow, 2012)

21. V. Shchukin, A. Halatov, Heat transfer, mass transfer and hydrodynamics of swirled flows in axisymmetric channels (Mashinostroenie, Moskov 1982)

22. A.Zuikov, G.Orekhov, T.Suehtina, MATEC Web of Conferences 193 (2018)

23. A. Zuikov, V. Orekhov, V. Volshanic, Vestnik MGSU, 5 150-161 (2013)

24. V. Volshanik, A. Zuikov, A. Mordasov. Swirling flow in hydraulic structures (Energoatomizdat, Moskov 1990) 